\title{
Accessing Emotion Patterns from Affective Interactions using Electrodermal Activity
}

\author{
Rui Henriques \\ Dep. of Computer Science and Eng. \\ IST, University of Lisbon \\ rmch@ist.utl.pt
}

\author{
Ana Paiva \\ GAIPS, INESC-ID and \\ IST, University of Lisbon \\ ana.s.paiva@ist.utl.pt
}

\author{
Claudia Antunes \\ Dep. of Computer Science and Eng. \\ IST, University of Lisbon \\ claudia.antunes@ist.utl.pt
}

\begin{abstract}
Measuring evocative emotions in affective interactions has become a critical step for effective engagements with computers. Electrodermal activity is believed to accurately isolate sympathetic responses, revealing paths to excitement, attention and arousal, and to differentiate emotional states. However, the inability to deal with varying amplitude and length of responses across individuals has led to its use as a simple intensity barometer. Thus, two questions remain unanswered. To which extent can electrodermal activity be used to recognize emotions? How do electrodermal responses vary between human-to-human and human-to-robot interactions?

To answer these questions, we propose a new method to mine the signal that surpasses the referred limitations, and conduct an extensive experiment to study the responses to emotion-evocative stimuli across different settings. Observations reveal emerging electrodermal patterns for each emotion and attractive accuracy levels for emotion recognition that increases when there is a link to the psychological traits of the subjects.
\end{abstract}

\section{INTRODUCTION}

Wrist-worn biometric sensors have been adopted to evaluate interactions by tracking user's emotional arousal via skin conductance, a form of electrodermal activity (EDA). Monitoring EDA is a simple, non-intrusive and able to isolate responses from the sympathetic nervous system [4].

Although a sympathetic response pattern is part of every emotion, the difficulty of normalizing the significantly different individual sympathetic responses led to its use basically as an intensity-axis marker. This is commonly accomplished by a combined analysis of basic features: level, response amplitude, rate, rise time and recovery time [4]. This analysis has a clear drawback - it discards flexible elicited behavior that can improve emotion recognition. For instance, the ability to mine expressive motifs sensitive to electrodermal sub-peaks holds the potential to separate anger from fear responses [1].

Thus, the target research question is: what is the impact of adopting more flexible approaches to mine electrodermal activity for emotion recognition in affective engagements? This question leads us to two other critical questions, also covered in this work. First, to which extent can we isolate electrodermal patterns for different emotion-evocative stimuli? Second, how do emotional responses in human-to-robot contexts compare with human-to-human contexts? In this work, we conduct an experimental study to answer these questions.

This paper is structured as follows. In section 2, relevant work on the use of biometric sensors in emotion-centered studies is covered. Section 3 describes the target experimental setting. Section 4 describes the proposed methodology to analyze the collected data. Finally, Section 5 presents the main results and synthesizes their main implications.

\section{CONTEXT}

Monitoring physiological signals is increasingly necessary to derive accurate analysis from affective interactions. Their use surpasses social masking, context-sensitivity of imageaudio monitoring, and track cognitive and sensitive emotional changes that are less obvious to perceive. However, their subtle, complex, variable and subjective manifestation and expression within and among individuals pose key challenges.

In the context of human-to-robot interaction, emotion recognition from physiological signals has been driven by responses to robot motion [8][9]. In virtual scenarios, feature-based learners were used to recognize emotions (engagement, anxiety, boredom, frustration and anger) from signals collected during parametrized game play-sessions [15]. Additionally, affective recognition has been largely applied in social and human-computer interaction [14]. Although the use signals to monitor interactions is increasingly accomplished [6], there is still a gap on how to describe and recognize physiological responses from emotion-evocative stimuli.

Good surveys that combine multiple physiological signals for emotion recognition include [7][17]. This work targets the electrodermal signal and exploits the boundaries of how much affective information can be disclosed by its analysis.

Electrodermal activity (EDA) is an electrical change in the skin that varies with the activation of the sympathetic nervous system, which is responsible for activating excitement and anticipation, and to mobilize the body's fight-or-flight action by mediating the neuronal-hormonal response [1]. Electrical changes in the skin are a result of an increased emotional arousal or cognitive workload that leads to an intense physical exertion and to sweating.By monitoring EDA is possible to detect periods of excitement, stress, interest and attention. However, heightened skin conductance is also related with engagement, hurting, intrigue, distress and anticipation [1] "the unknown behind the wall" - an inhibition response to punishment, avoidance or frustrative non-reward, which are different forms of anxiety [4]. These recent clarifications claim for more robust experimental settings and mining methods. 


\begin{tabular}{ll}
\hline & Experiments with brief and isolated stimuli include the study of: \\
innocence using the guilty test; familiarity using meaningful and \\
unfamiliar stimuli ; relevance through non-balanced occurrence of a \\
stimuli & $\begin{array}{l}\text { category or elicitation of priorities; affective valence (there is not yet } \\
\text { strong discrimination evidence); and planning and decision-making } \\
\text { processes via the "somatic marker" hypothesis. Backward masking of } \\
\text { conditioning stimulus is often used to prevent its conscious recognition. }\end{array}$ \\
\hline \multirow{5}{*}{ Continuous } & SCL and the frequency of spontaneous responses (nsSCR) are key \\
stimuli & measures. Experiments include the study of: strong emotions repro- \\
& acing, for instance, genuine states of fear (heightened SCL) and \\
anger (heightened nsSCR); reappraisal through authentic, forbidden \\
different forms of social interaction involving, for instance, judgment \\
(nsSCR inversely related to the judged permissiveness of a questioner), \\
distress invocation, or the contagious effect by relating, for instance, \\
heightened SCL with interacting with over-involved individuals. En- \\
ergy mobilization seems to be the driver for tasks that either require \\
an effortful attention or invoke the concepts of stress and affect.
\end{tabular}

TABLE I: Implications of emotion-centered EDA studies [6][4][1]

Advantages of measuring EDA include sympatheticcentered response, trial-by-trial visibility, significance of individual differences (reliably associated with psychopathological states), and its simple discrimination after a stimulus presentation. However, EDA has a relatively slow-moving response (latency of the elicited response and tonic shifts varying among individuals [4]), requires lengthy warm-up periods, and has multiple influences that may be either related with the personal attention and significance or with stimuli activation.

\section{A. Setting the approach}

Evaluation of affective interactions aims to achieve reliable emotion recognition rates. The key implication is to gain access to someone's feelings. For this purpose, according to the model proposed in [6], 4 questions need to be answered.

First, which approach to follow? User dependency, stimuli subjectivity and analysis time are the key axes. We target userindependent, non-subjective and dynamic evaluations.

Second, which models of emotions select? Applied models include the target discrete model centered on 6-to-8 categories, the dimensional valence-arousal, among others, such as Ellsworth's agency and Weiner's attributions [6].

Third and fourth questions - Which experimental conditions to adopt? Which data processing and mining techniques to adopt? - will be respectively assessed in sections 3 and 4 .

\section{B. Related work on EDA and emotions}

Electrodermal phenomena can be understood by discovering tonic changes (SCL) related with general states of arousal and alertness, and phasic changes (SCR) related with attentional processes (novelty, intensity, and significance). Historical EDA studies have been focused on learning efficiency, response speed and, as target by this paper, emotional appraisal. The implications of these studies are synthesized in Table I.

\begin{tabular}{|c|c|}
\hline $\begin{array}{l}\text { Pre- } \\
\text { processing }\end{array}$ & $\begin{array}{l}\text { Goal: remove contaminations (noise, ext. interferences and artefacts). } \\
\text { Methods: segmentation; discard of initial and end signal bands; smooth- } \\
\text { ing filters; low-pass filters such as Adaptive, Elliptic or Butterworth; } \\
\text { baseline subtraction; normalization; and discretization techniques. }\end{array}$ \\
\hline $\begin{array}{l}\text { Feature } \\
\text { extraction }\end{array}$ & $\begin{array}{l}\text { Goal: extract expressive features - statistical (mean, deviation), tem- } \\
\text { poral (rise and recovery time), frequency- and temporal-frequent (ge- } \\
\text { ometric analysis, multiscale sample entropy, sub-band spectra). } \\
\text { Methods: rectangular tonic-phasic windows; moving and sliding fea- } \\
\text { tures (as mean, median and deviation); transformations (Fourier, } \\
\text { wavelet, empirical, Hilbert, singular-spectrum); principal, independent } \\
\text { and linear component analysis; projection pursuit; auto-associative } \\
\text { networks; multidim. scaling; and self-organizing maps. }\end{array}$ \\
\hline $\begin{array}{l}\text { Feature } \\
\text { selection }\end{array}$ & $\begin{array}{l}\text { Goal: remove features without significant correlation with the emotion } \\
\text { under assessment (to improve the space exploitation). } \\
\text { Methods: sequential forward/backward selection, sequential floating } \\
\text { search, branch-and-bound search, principal component analysis, Fisher } \\
\text { projection, classifiers (e.g. decision tress, bayesian networks), Davies- } \\
\text { Bouldin index, and analysis of variance methods. }\end{array}$ \\
\hline Recognition & $\begin{array}{l}\text { Goal: classify emotions using the selected features. } \\
\text { Methods: wide-variety of deterministic and probabilistic learners } \\
\text { including: k-nearest neighbours, regression trees, random forests, } \\
\text { Bayesian networks, support vector machines, canonical correlation } \\
\text { and linear discriminant analysis, neural networks, and Marquardt-back } \\
\text { propagation. }\end{array}$ \\
\hline
\end{tabular}

TABLE II: Methods to mine physiological signals [10][7]

\section{Related work on mining EDA}

Common approaches rely on the features extracted from the signal, neglecting its motifs. For discrete stimuli, the response amplitude is a key feature. Response latency, rise time and half recovery time are sporadically adopted, although their relation to psychophysiological processes remains yet unclear [4]. For prolonged stimulation, SCL and both specific responses (SCR) and spontaneous responses (nsSCR) are considered and computed using different minimum amplitudes. The analysis of traits commonly recurs to nSSCR, SCL, response amplitude and habituation. The challenge is on deciding whether to use or not a range correction, by capturing extreme EDA values during a session [4]. Psychometric principles and surveys are additionally adopted to view EDA response as a trait [4].

More advanced techniques to pre-process and mine generic signals have also been applied [10][7]. These techniques can be divided in four steps and are synthesized in Table II.

Recent work shows that the use of sequential data mining techniques, when preceded by a symbolic approximation, can reveal additional expressive behavior of the signal [6].

\section{DATA COLlection}

To study electrodermal response to discrete emotionevocative stimuli, we conducted a tightly-controlled labexperiment. The stimuli were carefully chosen with the support of experts to evoke similar emotional responses across humanto-human and human-to-robot settings. Eight different stimuli, 5 emotion-centered stimuli and 3 others (captured during periods of strong physical effort, concentration and resting), were presented to each subject. The target discrete emotions ${ }^{1}$ are empathy (following common practices in speech tone and body approach [16]), expectation (possibility of gaining an additional reward), positive-surprise (unexpected attribution of a significant incremental reward), stress (impossible riddle to

\footnotetext{
${ }^{1}$ scripts available in http://web.ist.utl.pt/rmch/research/software/eda
} 


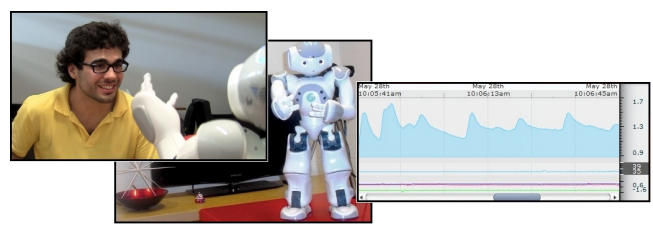

Fig. 1: Experimental setting (human-to-robot scenario)

solve in a short time to maintain the incremental reward) and frustration (self-responsible loss of the initial and incremental rewards). Emotional responses are averagely framed within 1030 seconds window for the selected stimuli. A time window of 6-8 minutes was provided between two stimulus to minimize influence, neutralize the subject emotional state, stabilize the signal, and remove the stress related with the experimental expectations.

30 participants, with ages between 19 and 24 (average of 21 years old), were randomly divided in two groups, $\mathrm{H}$ and R. Signal collections from both groups were intertwined to reduce bias from external factors. Participants from group $\mathrm{H}$ interacted with an human agent, an actor with a structured and flexible script. Subjects from group $\mathrm{R}$ interacted with a robot. From the subjects that participated in the experiment, 26 subject's collections were considered valid ${ }^{2}: 13$ from group $\mathrm{H}$ (5 women and 8 men) and 13 from group $\mathrm{R}$ ( 5 women and 8 men). The resulting dataset contains above 200 collected EDA signals ${ }^{3}$, which satisfies the statistical requirements for the mining methods proposed in the next section.

The robot used for this experience was $\mathrm{NAO}^{4}$. The behavior of NAO was expressively implemented and affective synthesized speeches recorded according to a flexible script similar to the one given to the human agent. The human-to-robot scenario was conducted as a wizard-of-Oz, with conditions carefully obeying to the requirements of this setting.

A 30-minutes warm-up period was included where subjects solved tests requiring a medium-to-high level of attention.

The adopted reward for all subjects was a pair of cinema tickets-offer and the potential additional reward was a Nintendo-Wii. The stimulus was conceived to evoke similar emotional reactions across individuals and it was presented in the same order in every experience.

An additional set of key practices was undertaken [13][3]: the states of very high and very low arousal were captured to normalize the features; the experiment was conducted in an appealing context to not desensitize the subject; the experience was recorded and documented to be audited and reproduced.

The collected EDA signals were obtained using wrist-worn Affectiva-QSensors ${ }^{5}$ and closely-controlled procedures.

Additionally, the following signals were collected using Affectiva technology: facial expression (for post-experimental validation and interpretation), skin temperature (to correct individual reactions to room temperature), and three-directional body-motion (to smooth correlated EDA variations).

\footnotetext{
${ }^{2}$ a baseline level of skin-conductivity above $0.5 \mu \mathrm{s}$

${ }^{3}$ raw signals available in http://web.ist.utl.pt/rmch/research/software/eda

${ }^{4}$ http://www.aldebaran-robotics.com

${ }^{5}$ http://www.affectiva.com/q-sensor/
}

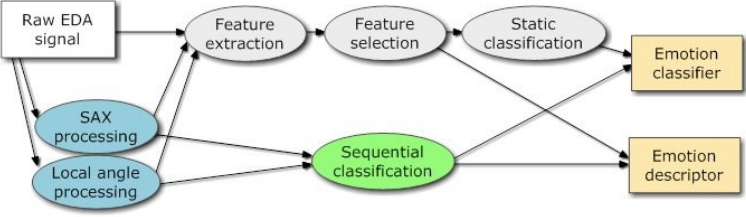

Fig. 2: Proposed FineAlly methodology to mine EDA responses

Finally, a survey was undertaken to categorize individuals according to the Myers-Briggs type indicator ${ }^{6}$. The results were used with the aim of studying meaningful correlations between the user's profile and emotional EDA responses.

\section{Computational Methodology}

This section describes a novel method, "FineAlly", for emotion recognition and description from $\mathrm{EDA}^{7}$. Fig.2 illustrates its main steps. Emotion recognition combines the traditional feature-based classification with the results provided from sequence learners and is centered on two expressive representations. The adopted representations either rely on: $i)$ SAX to normalize individual differences while still preserving overall response pattern, or on ii) local angles to enhance the local sub-peaks of a response. Additionally, emotion characterization is accomplished using both feature-based descriptors and the transition lattices generated by sequence learners.

\section{A. Processing the signal using $S A X$}

We propose the use of Symbolic ApproXimation (SAX) [11] to normalize the differences of EDA responses across individuals. SAX supports a reduced and normalized cardinality (range of values) and dimensionality (number of time points), and a lower-bounding guarantee by transforming real-valued time series into a symbolic representation. SAX allows a time series of arbitrary length $n$ to be mapped into a string of $w$ length $(w<n$, typically $w \ll n)$ with an alphabet size $d>2 \in \mathbb{N}$. Different criteria may be adopted to fix the signal cardinality and dimensionality: with respect to all stimulus, to a target stimuli, to all subjects and to a scenario (either human-tohuman or human-to-robot). SAX behavior can be synthesized in two steps. First, the signal is transformed into a Piecewise Aggregate Approximated (PAA) representation. Second, the PAA signal is symbolized into a discrete string allowing lower bounding. A Gaussian distribution is used to produce symbols with equiprobability from statistical breakpoints [11].

\section{B. Processing the signal based on local angles}

Although normalization of cardinality is needed, it may smooth relevant sub-peaks. Additionally, the traditional criteria to find sub-peaks using amplitude-thresholds is not sensitive to individual differences [7]. For this, we propose a representation to enhance local variations. Relying on parametrized time-windows, the angle for the electrodermal variation is computed, and it is translated into a symbol based on the target number of symbols. Similarly to SAX, the angle

\footnotetext{
${ }^{6}$ tests available in http://web.ist.utl.pt/rmch/research/software/eda

${ }^{7}$ software available in http://web.ist.utl.pt/rmch/research/software/eda
} 
break points are also defined assuming a Gaussian distribution. The following SAX representation for EDA over a short time interval: $\langle 17,13,15,14,18,19,16,14,13,12,16,16\rangle$, would be translated into the following angle representation: $<0,4,1,5,5,0,1,1,1,5,4>$ when adopting an 6-dim alphabet.

\section{Mining the signal using feature-based classifiers}

The features are extracted recurring to two methods: $i$ ) computation of basic metrics over raw and SAX representations (SCL, nsSCR, amplitude, rising and recovery time), and ii) statistical and geometric features retrieved from all representations (including dispersion metrics as deviation and distortion; centroid metrics; among others metrics as the Pearson product-moment, the kurtosis or the gradient).

The feature selection is performed recurring to two techniques: i) statistical analysis of variance (ANOVA) to understand the impact of the feature on predicting an emotion (using SPSS software), and ii) combined influence from multiple attribute selection techniques (using WEKA [5]).

Finally, feature-based classifiers (from WEKA) are applied.

\section{Mining the signal using hidden Markov models}

Sequence classifiers are applied to consider more flexible electrodermal behavior. Popular sequence learners include recurrent neural networks and dynamic Bayesian networks [2]. This paper adopts hidden Markov models (HMM) due to their stability, simplicity and flexible parameter-control [12]. Given a set of signals labeled with a specific emotion, the core task is to learn the generation and transition probabilities of a hidden automaton (that follows a Markov constraint) per emotion. This is done by maximizing a likelihood function using an efficient forward-backward algorithm until convergence [12][2]. Finally, given a non-labeled signal, emotions can be naively selected by evaluating their generation probability over the learned automaton. For this purpose, the Viterbi learning setting is applied. An additional exploitation of the lattices for each emotion can be used to retrieve emerging patterns.

We propose a parameterization of HMMs based on the signal properties (e.g. high dimensionality leads to an increased number of hidden states). Additionally, we adapted the HMMs to deal with multiple EDA representations by computing the joint probability of the paths learned for each representation.

\section{RESUlts}

\section{A. Observations from Feature Analysis ${ }^{8}$}

1) EDA features and affective responses: To understand how can the extracted features differentiate the emotions under assessment, the one-way ANOVA test was applied with the Tukey post-hoc analysis. A significance level of 5\% was consider for the Levene's test of variance homogeneity, ANOVA and Tukey tests. Both EDA metrics and statistical features derived from the raw and processed signals were considered.

Results, synthesized in Table III, show a representative set of features able to differentiate emotions ${ }^{8}$. Dispersion metrics to discriminate positive emotions and gradient plus centroid

\footnotetext{
${ }^{8}$ statistical sheets available in http://web.ist.utl.pt/rmch/research/software/eda
}

\begin{tabular}{lll}
\hline $\begin{array}{l}\text { Features (with strongest statistical signif- } \\
\text { icance to differentiate emotions' sets) }\end{array}$ & Separated emotions & Interaction \\
\hline $\begin{array}{l}\text { Accentuated dispersion metrics (as the } \\
\text { mean root square error) from the SAX } \\
\text { and local-angle representations }\end{array}$ & $\begin{array}{l}\text { Positive (empathy, ex- } \\
\text { pectation, surprise) }\end{array}$ & Human-robot \\
\hline $\begin{array}{l}\text { Recovery time, median (relevant to quan- } \\
\text { tify the sustenance of peaks) and distor- } \\
\text { tion from SAX signals }\end{array}$ & $\begin{array}{l}\text { Positive from negative } \\
\text { from neutral emotions }\end{array}$ & Human-robot \\
\hline $\begin{array}{l}\text { Gradient (revealing long-term sympa- } \\
\text { thetic activation by measuring the EDA } \\
\text { baseline changed) and centroid metrics }\end{array}$ & Fear from frustration & Overall \\
\hline Rise time & Empathy from others & Human-robot \\
\hline Response amplitude & Surprise from others & Human-robot \\
\hline
\end{tabular}

TABLE III: EDA features with potential to discriminate emotions

\begin{tabular}{ll}
\hline Origin & Correlations with higher statistical significance \\
\hline $\begin{array}{l}\text { Local-angle } \\
\text { features }\end{array}$ & $\begin{array}{l}\text { Dispersion metrics positively corr. with the felt intensity, the understand- } \\
\text { ing of the agent's intention, and his level of influence on felt emotions. }\end{array}$ \\
\hline $\begin{array}{l}\text { SAX-based } \\
\text { features }\end{array}$ & $\begin{array}{l}\text { Dispersion metrics positively corr. with the felt stimulation, and the } \\
\text { perceived empathy, trust and confidence of the agent. }\end{array}$ \\
\hline $\begin{array}{l}\text { Computed } \\
\text { metrics }\end{array}$ & $\begin{array}{l}\text { Amplitude posit. corr. with the perceived agent's influence on emotions; } \\
\text { Amplitude negatively corr. with the felt pleasure; }\end{array}$ \\
Rise time neg. corr. with the perceived positivism on the agent's attitude.
\end{tabular}

TABLE IV: Illustrative EDA features that may complement surveys

metrics to discriminate negative emotions. Additionally, features can be used to isolate specific emotions (as rise time and response amplitude) or to predict the affective valence (as recovery time, median and distortion). Other metrics as kurtosis (revealing the flatness of the response's major peak) and metrics derived from the temperature signal were also able to differentiate with significance some of the target emotions.

2) EDA features and the experimental conditions: EDA can be additionally adopted to complement survey assessments. For this purpose, bivariate Pearson correlation (between scale variables assessed in the final survey and EDA metrics) was performed using a 5\% significance level. Table IV synthesizes the most significant correlations found. They include positive correlation of local-angle dispersion (revealing changes in the EDA's gradient) with intensity, perceived intention and felt influence; positive correlation of SAX-based dispersion (revealing heightened variations from the baseline) with the perceived empathy, trust and confidence; higher amplitude of responses for heightened felt influence and lower pleasure; and quicker rise time for heightened perceived optimism.

Finally, features derived from the overall signal collected by each subject (without stimuli-partitions) were found to be correlated with the adopted experimental condition (human-tohuman vs. human-to-robot). To assess the influence of the experimental setting in the signal we performed a t-test. Results from the use of SAX-based signals show that human-to-human interactions (in comparison to human-to-robot interactions) have significantly: $i$ ) higher values of dispersion and kurtosis (revealing heightened emotional response), and ii) a higher median (revealing an increased ability to sustain peaks).

3) EDA features and the subject profile: Pearson correlations were also used to relate EDA with the subjects profile. 


\begin{tabular}{ll}
\hline Myers-Briggs type & Correlated features $([+]$ positive corr.; $[-]$ negative corr.) \\
\hline \multirow{3}{*}{ Extrovert-introvert } & $\begin{array}{l}{[+] \text { Dispersion metrics of SAX-based signal }} \\
{[-] \text { Centroid metrics of SAX-based signal }} \\
{[-] \text { Response amplitude }}\end{array}$ \\
\hline \multirow{3}{*}{ Sensing-intuition } & {$[-]$ Dispersion metrics of raw and SAX-based signal } \\
& {$[-]$ Dispersion metrics of local-angles } \\
& {$[-]$ Rise time } \\
\hline \multirow{3}{*}{ Feeling-thinking } & {$[+]$ Median and dispersion metrics of SAX-based signal } \\
& {$[-]$ Declive and centroid metrics of local-angles } \\
& {$[-]$ Rise time } \\
\hline \multirow{3}{*}{ Judging-perceiving } & {$[-]$ Centroid metrics of raw signal } \\
& {$[-]$ Dispersion metrics of SAX-based signal } \\
& {$[+]$ Response amplitude }
\end{tabular}

TABLE V: Influence of subjects' profile on EDA responses

This analysis is key to turn emotion-driven learning sensitive to psychophysiological traits of the subject under assessment. A positive/negative correlation means that a polarization towards either the extrovert, sensing, feeling or perceiving type is related with higher/lower values for the target feature.

We can see, for instance, that extroverts have a more instable signal (higher dispersion) although less intense (lower amplitude), while sensers and feelers' responses are quicker. Table V synthesizes the significant correlations.

\section{B. Emotion Recognition}

The recognition score is accuracy, the ability to correctly label an unlabeled signal (i.e. to identify the underline emotion from 5 emotions). Accuracy was computed using a 10 crossfold validation over the collected 234 signals.

1) Feature-based recognition: Previously, we found that some statistical features and basic metrics are significantly able to differentiate groups of emotions. Now, we evaluate to which extent can a set of classifiers using the extracted features correctly label the emotion of an unlabeled EDA response. Despite the observed accuracy is lower than the 80\% threshold achieved in state-of-the-art studies [7][17], our study does neither combine a wide-variety of signals nor rely on strong imagery and audio suggestions.

Fig.3 synthesizes the results from feature-driven classification. The isolated use of EDA metrics and of features from SAX or local angles leads to an accuracy near $50 \%$. However, their integration leads to levels near $60 \%$. Additionally, accuracy increases when skin temperature is included. The best classifiers are the logistic-based learners, followed by random forests. Logistic-based learners use regressions on the real-valued features to affect the probability score of each emotion. However, when no feature selection method is applied, Bayesian nets are the best alternative.

Despite the difference between human-to-human and human-to-robot scenarios, classifiers are still able to recognize emotions when mixing the cases. For instance, C4.5 trees have dedicated branches for each scenario, while IB $k$ usually selects the features from a sole scenario when $k<4$.

2) Sequential-based recognition: Table VI shows that when considering sequential behavior, the accuracy levels can rise nearly 10pp. An intricate observation from these results was

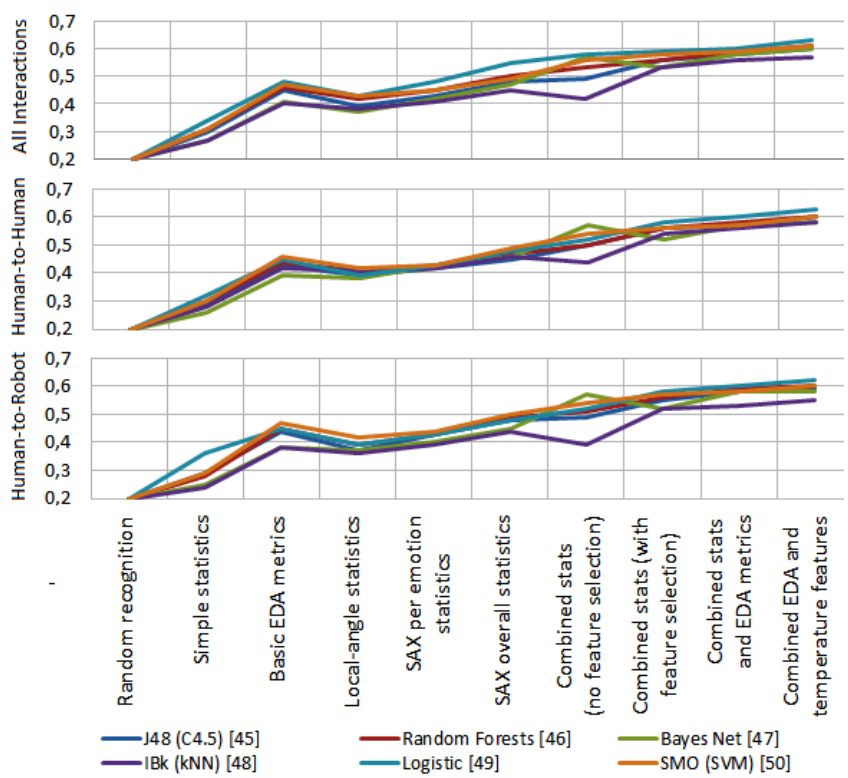

Fig. 3: Feature-driven recognition accuracy of 5 emotions

\begin{tabular}{|c|c|c|c|c|c|c|}
\hline & & & 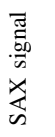 & 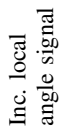 & 莺 & 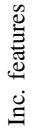 \\
\hline \multirow{6}{*}{$\begin{array}{l}\text { HMM (fully } \\
\text { connected } \\
\text { architecture) }\end{array}$} & \multirow{3}{*}{$\begin{array}{l}\text { Recognition } \\
\text { accuracy }\end{array}$} & All & 0.40 & 0.42 & 0.46 & 0.67 \\
\hline & & Robot & 0.39 & 0.41 & 0.44 & 0.66 \\
\hline & & Human & 0.39 & 0.42 & 0.45 & 0.67 \\
\hline & \multirow{3}{*}{$\begin{array}{l}\text { Discrimination } \\
\text { accuracy }\end{array}$} & All & 0.86 & 0.88 & 0.89 & - \\
\hline & & Robot & 0.87 & 0.88 & 0.91 & - \\
\hline & & Human & 0.86 & 0.88 & 0.90 & - \\
\hline \multirow{6}{*}{$\begin{array}{l}\text { HMM } \\
\text { (left-to-right } \\
\text { architecture) } \\
{[12]}\end{array}$} & \multirow{3}{*}{$\begin{array}{l}\text { Recognition } \\
\text { accuracy }\end{array}$} & All & 0.43 & 0.44 & 0.48 & 0.71 \\
\hline & & Robot & 0.42 & 0.43 & 0.47 & 0.71 \\
\hline & & Human & 0.41 & 0.44 & 0.47 & 0.69 \\
\hline & \multirow{3}{*}{$\begin{array}{l}\text { Discrimination } \\
\text { accuracy }\end{array}$} & All & 0.87 & 0.88 & 0.90 & - \\
\hline & & Robot & 0.87 & 0.89 & 0.90 & - \\
\hline & & Human & 0.87 & 0.88 & 0.89 & - \\
\hline
\end{tabular}

TABLE VI: Accuracy of sequence learners to recognize an emotion (out of 5) and to correctly discard the 3 least probable emotions

the high sensitivity of the learned HMMs to accurately neglect 3 emotion labels that do not fit in the learned behavior. The challenge is to generalize the multiple subject behavior per emotion. One solution is either to select representative signals through a cluster analysis or to embrace the error and use HMMs as a discriminator to discard non-fitting responses.

Constrained HMMs, as left-to-right architectures, can improve accuracy. This is done by controlling the state transitions through the values of the initial lattice.

Although the local-angle representation is not as critical as SAX representations, its weighted use also impacts accuracy.

Finally, when combining the sequential classifiers with the output of probabilistic feature-based classifiers (logistic-based learners were the choice), the accuracy increases.

The why behind these scores resides on the ability of HMMs to: $i$ ) detect expressive behavior as peak-sustaining values and fluctuations (hardly measured by features); ii) to cope with SCL differences (with the SAX scaling strategy being 
done with respect to all stimulus, to the target stimuli, to all subjects or to subject-specific responses); iii) to cope with dimensionality differences; iii) to cope with SCR differences using the local-angle representation; and $i v$ ) to deal with lengthy responses (by increasing the number of hidden states). Additionally, by controlling cardinality using SAX: HMMs can capture either a smoothed behavior (alphabet size less than 8) or a more delineated behavior (alphabet size above 10).

3) Profile inclusion: The insertion of the relative score for the four Myers-Briggs types was found to increase the accuracy of IBk, who tend to select responses from subjects with related profile. Motivated by this observation, we adopt a strategy for the non-lazy probabilistic learners by creating four partitions of the data, with the first separating extroverts from introverts and so on, and by learning one model for each profile. Recognition for a test instance now relies on the equally weighted combined output of each model, which result in an increased accuracy of 1-3pp. Although the improvement seems to be subtle, it is in fact promising when taking into consideration the significant lower size of the training datasets used to learn the models for each personality type.

\section{Emotion Response Description}

Along with emotion recognition, the characterization of EDA responses to emotion-evocative stimuli is of key value to understand psycho-physiological drivers of the sympathetic system and to develop rule-based systems to access emotional states. A simple way to describe emotion patterns is to use plots with the most discriminative features (see Table III). We additionally propose the use of HMM lattices as a pattern descriptor. Distance metrics over the learned lattices revealed statistically significant differences across the emotions and the interaction settings. This evidence is supported by the recognition scores disclosed in the previous section.

\section{Implications}

Previous observations reveal that features extracted from the (pre-processed) signals can be adopted to isolate emotions across different interaction settings (Table III). Classifiers that combine feature-based and sequential learning achieve optimum accuracy rates. Their underlying learned models can be used to characterize emotional responses. Finally, EDA was observed to be correlated with psychological traits (Table V), which can be used to guide emotion recognition.

\section{CONCLUSION}

This work provides two major contributions. First, we propose a new methodology, FineAlly, to mine electrodermal activity in emotion-centered interactions. This methodology is centered on expressive pre-processing steps followed by the joint application of feature-driven and sequential-based mining techniques. FineAlly overcomes the limitations of traditional methods to deal with expressive behavior and with individual response differences.

Second, this work reveals emerging properties from electrodermal responses related with different emotions and experimental settings. In particular, the recognition accuracy using solely the electrodermal signal is competitive with state-ofthe-art studies that combine multiple physiological signals.

Additionally, we showed that psychological traits can guide this task by correcting profile-driven differences, opening a new direction on how to measure affective interactions.

The quantitative assessment shows that emotion recognition significantly improves when adopting more flexible methods to mine the electrodermal signal. Additionally, feature correlation analysis and discriminative mining of generative models show meaningful differences among emotional stimuli and experimental settings. These answers to the target research questions trigger new implications not only for psychophysiology and neuroscience, but especially to human-robot and social interaction research.

\section{ACKNOWLEDGMENT}

The authors would like to thank: i) Arvid Kappas, ii) Tiago Ribeiro for implementing the NAO's behavior with sharp expressiveness, and iii) Iolanda Leite and Ivo Capelo for their support during the experiment preparation and execution.This work was supported by national funds through FCT (Fundação para a Ciência e a Tecnologia), under project PEst-OE/EEI/LA0021/2013, by the project EMOTE from the EU 7thFramework Program (FP7/20072013) under grant agreement n.317923, by the project D2PM (PTDC/EIAEIA/110074/2009) and under the PhD grant SFRH/BD/75924/2011.

\section{REFERENCES}

[1] J. Andreassi, Psychophysiology: Human Behavior And Physiological Response. Lawrence Erlbaum, 2007.

[2] C. M. Bishop, Pattern Recognition and Machine Learning (Inf. Science and Stat.). Secaucus, NJ, USA: Springer-Verlag New York, Inc., 2006.

[3] D. O. Bos, "Eeg-based emotion recognition the influence of visual and auditory stimuli," Emotion, vol. 57, no. 7, pp. 1798-806, 2006.

[4] J. Cacioppo, L. Tassinary, and G. Berntson, Handbook of psychophysiology. Cambridge University Press, 2007.

[5] M. Hall, E. Frank, G. Holmes, B. Pfahringer, P. Reutemann, and I. H. Witten, "The weka data mining software: an update," SIGKDD Explor. Newsl., vol. 11, no. 1, pp. 10-18, Nov. 2009.

[6] R. Henriques, A. Paiva, and C. Antunes, "On the need of new methods to mine electrodermal activity in emotion-centered studies," in $A A M A S^{\prime} 12$, 8th IW on ADMI. Springer-Verlag LNAI series, 2012.

[7] S. Jerritta, M. Murugappan, R. Nagarajan, and K. Wan, "Physiological signals based human emotion recognition: a review," in CSPA, 2011 IEEE 7th International Colloquium on, 2011, pp. $410-415$.

[8] D. Kulic and E. A. Croft, "Affective state estimation for human-robot interaction," Trans. Rob., vol. 23, no. 5, pp. 991-1000, Oct. 2007.

[9] I. Leite, R. Henriques, C. Martinho, and A. Paiva, "Sensors in the wild: Exploring electrodermal activity in child-robot interaction," in HRI. ACM/IEEE, 2013, pp. 41-48.

[10] C. S. Lessard, Signal Processing of Random Physiological Signals, ser. S.Lectures on Biomedical Eng. Morgan and Claypool Publishers, 2006.

[11] J. Lin, E. Keogh, S. Lonardi, and B. Chiu, "A symbolic representation of time series, with implications for streaming algorithms," in $A C M$ SIGMOD workshop on DMKD. NY, USA: ACM, 2003, pp. 2-11.

[12] K. Murphy, "Dynamic bayesian networks: Representation, inference and learning," Ph.D. dissertation, UC Berkeley, CS Division, 2002.

[13] P. Petrantonakis and L. Hadjileontiadis, "Emotion recognition from eeg using higher order crossings," TITB, vol. 14, no. 2, pp. 186-197, 2010.

[14] R. W. Picard, E. Vyzas, and J. Healey, "Toward machine emotional intelligence: Analysis of affective physiological state," IEEE Trans. Pattern Anal. Mach. Intell., vol. 23, no. 10, pp. 1175-1191, Oct. 2001.

[15] P. Rani, C. Liu, N. Sarkar, and E. Vanman, "An empirical study of machine learning techniques for affect recognition in human-robot interaction," Pattern Anal. Appl., vol. 9, no. 1, pp. 58-69, May 2006.

[16] P. Rani, N. Sarkar, C. A. Smith, and L. D. Kirby, "Anxiety detecting robotic system: towards implicit human-robot collaboration," Robotica, vol. 22, no. 1, pp. 85-95, Jan. 2004.

[17] J. Wagner, J. Kim, and E. Andre, "From physiological signals to emotions: Implementing and comparing selected methods for feature extraction and classification," in ICME. IEEE, 2005, pp. 940 -943. 